NASA TECHNICAL NOTE

\begin{tabular}{l}
\multirow{2}{*}{} \\
$\vdots$ \\
$\vdots$ \\
$\vdots$ \\
$\vdots$
\end{tabular}

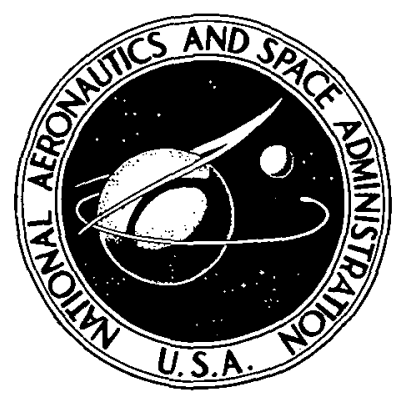

NASA TN D-7417

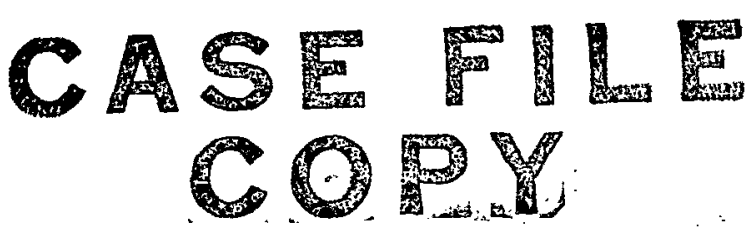

\title{
ULTRASONIC SIGNAL ENHANCEMENT BY RESONATOR TECHNIQUES
}

by Joseph S. Heyman

Langley Research Center

Hampton, Va. 23665 


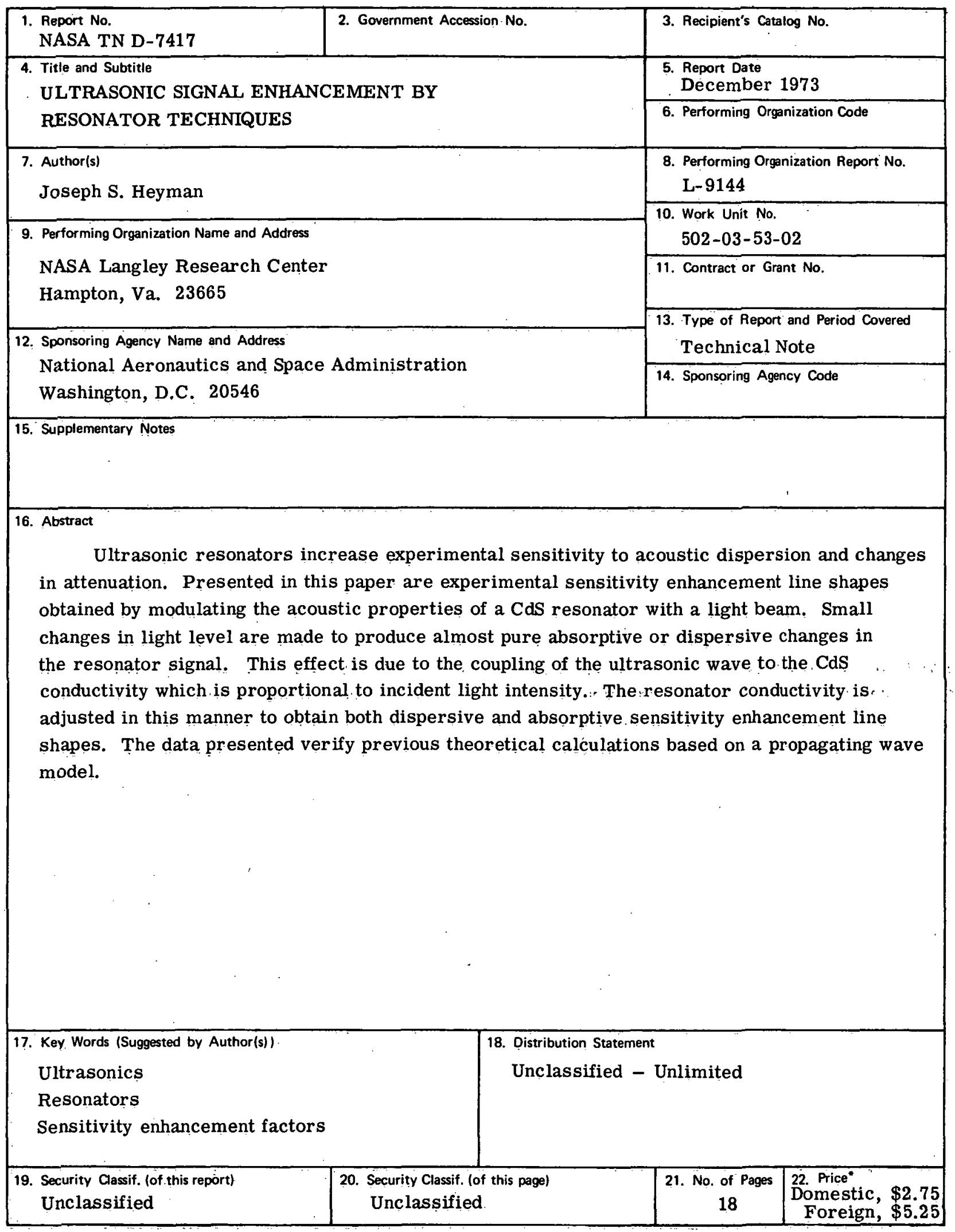

"For sole by the National Technical Information Service, Springfield, Virginia 22151 


\title{
ULTRASOONIC SIGNAL ENHANCEMENT BY RESONATOR TECHNIQUES
}

\author{
By Joseph S. Heyman
}

Langley Research Center

\section{SUMMARY}

\begin{abstract}
Ultrasonic resonators increase experimental sensitivity to acoustic dispersion and changes in attenuation. Presented in this paper are experimental sensitivity enhancement line shapes obtained by modulating the acoustic properties of a CdS resonator with a light beam. Small changes in light level are made to produce almost pure absorptive or dispersive changes in the resonator signal. This effect is due to the coupling of the ultrasonic wave to the CdS conductivity which is proportional to incident light intensity. The resonator conductivity is adjusted in this manner to obtain both dispersive and absorptive sensitivity enhancement line shapes. The data presented verify previous theoretical calculations based on a propagating wave model.
\end{abstract}

\section{INTRODUCTION}

Resonators are widely used in ultrasonics to improve the measurement sensitivity to changes in absorption and dispersion in acoustic material. (See ref. 1.) These changes are due to many different interactions in the resonator such as nuclear acoustic resonance (NAR), Alpher-Rubin effect, phonon coupling to the charge carrier system, acoustic paramagnetic resonance (APR), and many others. (See ref. 2.) Even though there exists a variety of physical interactions producing these changes, the role of the resonator in each case is the same. It is therefore important to have a theoretical understanding and an experimental verification of the part that resonators play in ultrasonic signal enhancement.

In this paper are presented experimental curves of the sensitivity enhancement line shapes. These line shapes are predicted from a theoretical analysis following a model described in references 1 and 2. The experimental procedure used in obtaining these measurements involves ultrasonic coupling to light-induced charge carriers in CdS. In this procedure, light incident on the CdS changes its conductivity and thus its ultrasonic properties. These effects are used to separate the absorptive and dispersive effects.

\section{SYMBOLS}

A particle velocity

$A_{1} \quad$ in-phase part of particle velocity 

$A_{2} \quad$ out-of-phase part of particle velocity
$\widetilde{A}$ complex particle velocity
a/2 sample length
$C_{A}$ absorption sensitivity enhancement factor for $A_{2}$
$C_{D}$ dispersion sensitivity enhancement factor for $A_{2}$
$G_{A}$ absorption sensitivity enhancement factor for $|A|$
$G_{D}$ dispersion sensitivity enhancement factor for $|A|$
I normalized light intensity
$\mathrm{K}^{2} \quad$ electromechanical coupling constant
k wave number
$\mathrm{k}_{\mathrm{m}} \quad$ wave number for " $\mathrm{mth}$ " mechanical resonance
$\mathrm{m}, \mathrm{n} \quad$ integers
Re real part of variable
$S_{A} \quad$ absorption sensitivity enhancement factor for $A_{1}$
$S_{D}$ dispersion sensitivity enhancement factor for $A_{1}$
t time
v ultrasonic phase velocity
$\mathbf{x}$ distance from transducer to a point in crystal
a characteristic attenuation per unit length
$\epsilon$ dielectric constant 
ultrasonic wavelength

$\sigma$

conductivity

$\tau$

round-trip reflection time for an ultrasonic wave in sample

${ }^{\tau} \mathbf{d} \quad$ transmitter gate offtime

$\psi$

ratio of dielectric relaxation frequency to ultrasonic frequency

$\omega$

$2 \pi$ times ultrasonic frequency

$\omega_{c} \quad 2 \pi$ times dielectric relaxation frequency

${ }^{\omega} \mathrm{m}$

$2 \pi$ times ultrasonic frequency corresponding to $\mathrm{mth}$ mechanical resonance

\section{THEORY}

The model used for this study is an idealized one-dimensional acoustic resonator. This model consists of a cylindrical resonator of length $a / 2$ with flat and parallel faces, one of which $(\mathrm{x}=0)$ is driven by a $\cos \omega t$ disturbance. This disturbance results in a damped traveling acoustic wave $\exp (-\alpha x) \cos (\omega t-k x)$ propagating in the material. In this equation, the characteristic attenuation per unit length is expressed by $a$, and the acoustic wave number is expressed by $k=\frac{2 \pi}{\lambda}=\frac{i \omega}{v}$ where $v$ is the acoustic phase velocity.

By assuming perfect reflection at the $x=a / 2$ face; the particle velocity $A$ at $x=0$ can be found by the superposition of all the partial waves present at $x=0$. It has been shown (refs. 1 and 2 ) that this condition results in the following equation:

$$
A=A_{1} \cos \omega t+A_{2} \sin \omega t
$$

where

$$
\begin{aligned}
& A_{1}=\frac{\exp (\alpha a)-\cos k a}{2(\cosh \alpha a-\cos k a)} \\
& A_{2}=\frac{\sin k a}{2(\cosh \alpha a-\cos k a)}
\end{aligned}
$$

The $A_{1}$ term is in phase with the driving oscillator whereas the $A_{2}$ term is advanced by $90^{\circ}$. 
If the assumption is now made that $a \mathrm{a}<<1$ and that measurements are made near mechanical resonance where $\mathrm{ka}=\mathrm{n} 2 \pi$, equations (1), (2), and (3) take the simplified form

$$
\begin{gathered}
|A|=\left(A_{1}^{2}+A_{2}^{2}\right)^{1 / 2}=\frac{1}{\left[(a a)^{2}+\left(k-k_{m}\right)^{2} a^{2}\right]^{1 / 2}} \\
A_{1} \approx \frac{a a}{(a a)^{2}+\left(k-k_{m}\right)^{2} a^{2}} \\
A_{2}=\frac{\left(k-k_{m}\right) a}{(a a)^{2}+\left(k-k_{m}\right)^{2} a^{2}}
\end{gathered}
$$

where $\mathbf{k}_{\mathrm{m}}=\frac{\omega_{\mathrm{m}}}{\mathrm{v}}$ and $\omega_{\mathrm{m}}=\frac{2 \pi \mathrm{mv}}{\mathrm{a}}$ related to the $\mathrm{mth}$ mechanical resonance. (See ref. 3.) As in references 1 and 2, an absorption sensitivity $S_{A}=\frac{\partial A_{1}}{\partial \alpha}$ and a dispersive sensitivity $S_{D}=\frac{\partial A_{1}}{\partial k}$ are defined with respect to $A_{1}$. Therefore, changes in $A_{1}$ due to small changes in a and $\mathrm{k}$ can be written as

$$
\Delta \mathrm{A}_{1}=\mathrm{S}_{\mathrm{A}} \Delta \alpha+\mathrm{S}_{\mathrm{D}} \Delta \mathrm{k}
$$

where

$$
\begin{aligned}
& S_{A}=\frac{a\left[a^{2}\left(k-k_{m}\right)^{2}-(a a)^{2}\right]}{\left[(a a)^{2}+\left(k-k_{m}\right)^{2} a^{2}\right]^{2}} \\
& S_{D} \approx \frac{-a\left[2 a a^{2}\left(k-k_{m}\right)\right]}{\left[(a a)^{2}+\left(k-k_{m}\right)^{2} a^{2}\right]^{2}}
\end{aligned}
$$

In a similar manner, an absorptive and dispersive sensitivity can be defined as $\mathrm{C}_{\mathrm{A}}=\frac{\partial \mathrm{A}_{2}}{\partial \alpha}$ and $\mathrm{C}_{\mathrm{D}}=\frac{\partial \mathrm{A}_{2}}{\partial \mathrm{k}}$, respectively. As in equation (7), small changes in $a$ and $\mathrm{k}$ will produce the following relations: 


$$
\Delta \mathrm{A}_{2}=\mathrm{C}_{\mathrm{A}} \Delta a+\mathrm{C}_{\mathrm{D}} \Delta \mathrm{k}
$$

where

$$
\begin{aligned}
& C_{A}=\frac{-a\left[2 a a^{2}\left(k-k_{m}\right)\right]}{\left[(a a)^{2}+\left(k-k_{m}\right)^{2} a^{2}\right]^{2}}=S_{D} \\
& C_{D}=\frac{a\left[(a a)^{2}-\left(k-k_{m}\right)^{2} a^{2}\right]}{\left[(a a)^{2}+\left(k-k_{m}\right)^{2} a^{2}\right]^{2}}=-S_{A}
\end{aligned}
$$

The last of the sensitivity enhancement line shapes to be considered here is defined with respect to $|A|$ and is, respectively, for absorption and dispersion $G_{A}=\frac{\partial|A|}{\partial a}$ and $\mathrm{G}_{\mathrm{D}}=\frac{\partial|\mathrm{A}|}{\partial \mathrm{k}}$. Similar to equations (7) and (10),

$$
\Delta|\mathrm{A}|=\mathrm{G}_{\mathrm{A}} \Delta a+\mathrm{G}_{\mathrm{D}} \Delta \mathrm{k}
$$

where

$$
\begin{aligned}
& G_{A}=\frac{-a^{2} a}{\left[(a a)^{2}+\left(k-k_{m}\right)^{2} a^{2}\right]^{3 / 2}} \\
& G_{D} \approx \frac{-a^{2}\left(k-k_{m}\right)}{\left[(a a)^{2}+\left(k-k_{m}\right)^{2} a^{2}\right]^{3 / 2}}
\end{aligned}
$$

Therefore, theoretical expressions for sensitivity enhancement line shape factors have been obtained. Each pair of factors $\left(S_{A}, S_{D} ; C_{A}, C_{D}\right.$; and $\left.G_{A}, G_{D}\right)$ are derived from an acoustic resonator signal (respectively, $A_{1}, A_{2}$, or $|A|$ ). The $A_{1}$ and $A_{2}$ factors are related by $S_{A}=-C_{D}$ and $C_{A}=S_{D}$ so that no new information is gained from $S_{D}$ and $C_{D}$ if $S_{A}$ and $C_{A}$ are known. 


\section{EXPERIMENTAL PROCEDURE}

Sensitivity enhancement values may be directly obtained by modulating a or $\mathrm{v}$ in an ultrasonic resonator and processing the detected radio frequency (RF) signal with a synchronous amplifier. The line shapes of these values are determined by sweeping the RF frequency through a mechanical resonance. The two sets of sensitivity enhancement line shapes $\left(G_{A}, S_{A}, C_{A}\right.$ and $\left.G_{D}, S_{D}, C_{D}\right)$ that are associated with a and $v$ are derived from $|A|, A_{1}$, and $A_{2}$ signals obtained with various detection techniques. In this section the manner in which this is accomplished is discussed.

A $10-\mathrm{MHz} \mathrm{x}$-cut quartz transducer is bonded to a flat and parallel thin $\left(10^{-3} \mathrm{~m}\right)$ sample of CdS. The crystal is high-purity grade-A photoconductive material from Eagle Picher Co. prepared so that longitudinal waves propagate along the direction of the " $\mathrm{C}$ " axis. In this study, an ultrasonic sampled continuous wave spectrometer (SCW of refs. 2 and 4) is used to excite the transducer-resonator as well as to measure the acoustic signal at the $x=0$ face.

In figure 1 is shown the experimental arrangement used for the measurements reported in this paper. (A very similar arrangement is the subject of NASA patent LAR 11435 that can be used as a calibration source for ultrasonics.) The continuous wave (CW) oscillator in this figure is gated on until ultrasonic saturation conditions occur in the sample. Then the transmitter is gated closed and the receiver gated open. The RF signal produced at the receiver amplifier is a measure of the decay of the ultrasonic waves in the sample. In contrast to a $\mathrm{CW}$ system, the measurement of the acoustic signal with an SCW system is made after its decay has begun. Therefore, the superposition of waves developed in reference 2 does not strictly apply here. The superposition theory sums up all waves present at the $x=0$ face just prior to the instant $\tau_{d}$ when the transmitter is gated off. Thus, it includes terms as

$$
\begin{aligned}
A= & \operatorname{Re} \tilde{A}=\operatorname{Re}(\exp (i \omega t)\{1+\exp [-(a a+i k a)] \\
& +\exp [-2(a a+i k a)]+\ldots\})
\end{aligned}
$$

At the instant $\tau_{d}$ the first term in the series vanishes. At a time $t=\tau+\tau_{d}$ (where $\left.\tau=\frac{a}{v}\right)$ the second term vanishes, and so on. It is still possible, however, to obtain a good agreement with the superposition theory by sampling an interval in the decay as close as possible to $\tau_{d}$ and by making the interval wider than several $\tau$. The early sampling does not let too many terms in the series "turn off" whereas the interval width averages 
the acoustic information during the interval. These conditions are achieved by sampling the decay at $t=\tau_{d}+2 \times 10^{-6}$ second and by using a detector time constant equal to about $4 \tau$. The resulting signal is a good approximation to theory.

The ultrasonic signal is preprocessed in several ways to obtain the desired line shape. A double balanced mixer and phase shifter are used to obtain $A_{1}$ and $A_{2}$ signals. For the $|\mathrm{A}|$ curves, a simple diode detector is used in place of the double balanced mixer. Once the desired signals are obtained by preprocessing, the resonator parameters are modulated. Care must be exercised in setting the modulation level. As can be seen in equation (7), for example, to measure $S_{A}$ with respect to $\Delta A_{1}$ it is necessary for $\triangle \alpha>\Delta k$ since $S_{A}$ and $S_{D}$ are similarly bound. This condition is possible by modulating the conductivity of the $\mathrm{CdS}$ resonator.

The acoustic absorption and dispersion for this material as a function of conductivity are developed in reference 5 . It is shown that for this case the absorption and velocity take the following form:

$$
\left.\begin{array}{c}
a=\mathrm{k} \frac{\mathrm{K}^{2}}{2} \frac{\psi}{1+\psi^{2}} \\
\mathrm{v}=\mathrm{v}_{0}\left(1+\frac{\mathrm{K}^{2}}{2} \frac{1}{1+\psi^{2}}\right)
\end{array}\right\}
$$

where $\psi=\frac{\omega_{c}}{\omega}, \quad v_{0}=4.4 \times 10^{5} \mathrm{~cm} / \mathrm{sec}$ is the phase velocity at $\omega_{\mathrm{c}}<\omega\left(\omega_{\mathrm{c}}=\frac{\sigma}{\epsilon}\right.$, the conductivity divided by the dielectric constant), and $\mathrm{K}^{2}$ is the electromechanical coupling coefficient $(\approx 0.02)$. Since CdS is a photoconducting material, $\sigma$ is proportional to $I$, the light intensity incident on the crystal. Under these conditions the effect of light on the acoustic parameters for a similar sample is shown in figure 2. (See ref.6.) It can be seen from this figure that a small change in the light intensity will produce a small change in the acoustic absorption and dispersion. If $\mathrm{I}=1$, then for small $\Delta \mathrm{I}, \Delta \mathrm{k} \gg \Delta \alpha$ and a signal related to dispersion is obtained. If, on the other hand, I $₫ 0.2$, then $\Delta a>\Delta \mathrm{k}$ and a signal related to absorption is obtained. The low-frequency audio oscillator is set to produce a small modulation $(\Delta \mathrm{I})$ in the light intensity and therefore a $\Delta \omega_{\mathrm{c}}$ in the sample. (See fig. 1.) This condition causes a modulated acoustic signal which is detected by a synchronous amplifier. By plotting the synchronous amplifier output as a function of the driving oscillator frequency, one obtains the sensitivity enhancement line shapes. 


\section{EXPERIMENTAL RESULTS AND DISCUSSION}

Shown in figures 3 and 4 are the experimental normalized curves for $|A|, A_{1}$, and $A_{2}$. Experimental values for $a, a$, and $\omega_{m}$ are used with equations (4), (5), and (6) to calculate the theoretical points shown in these figures with $a=0.6 \mathrm{~cm}^{-1}$ for figure 5 (b) and $a=0.1$ for the rest of the figures. The $|\mathrm{A}|$ curve is corrected for nonlinearity in the diode detector. Figure 4 shows the in-phase and out-of-phase components of $|\mathbf{A}|$. The slight deviation in these curves noticeable on the high-frequency side may be attributed to inhomogeneous responses caused primarily by nonuniform mobility or nonuniform production of photoinjected carriers. This condition results in a nonuniform absorption and dispersion of the acoustic wave which appears to be similar to nonparallel resonator faces. Nonparallel faces produce a frequency doublet favoring in amplitude, the first of the appearing resonances. (See ref. 3.) Thus, a slight nonparallelism results in a small "bump" on the high-frequency side of the mechanical resonance. No "bump" occurs in the resonance curves from unilluminated conditions. Furthermore, to rule out nonuniformity of the light beam as a cause, the introduction of a diffuser into the defocused beam between the sample and the lens has little effect on the curve shape. Thus, it appears that the apparent inhomogeneous response is due to nonuniformities in the sample.

Shown in figures $5(\mathrm{a})$ and $5(\mathrm{~b})$ are the sensitivity enhancement line shapes $\mathrm{G}_{\mathrm{A}}$ and $G_{D}$ described by equations (14) and (15). These curves are obtained from the diode detector used with the $|\mathrm{A}|$ curve. It was experimentally determined that the corrections applied to the $|A|$ curve would not have to be applied to the $G_{A}$ or $G_{D}$ curve. It was found that less than a 5-percent error in signal amplitude occurred down to 20 percent of peak value. In figure $5(\mathrm{~b})$, the line shape $G_{D}$ is obtained with the maximum light intensity used. The inhomogeneous effect on the high-frequency side is more pronounced here in concurrence with the attributed cause.

The remaining figures are taken with the double balanced mixer. In figure 6 is shown the line shape $S_{A}$ of equation (8) which is equal to the $-C_{D}$ line shape in equation (12). In figure 7 the $C_{A}$ line shape is shown. One significance of these line shapes is their zero crossings, one for the $C_{A}$ and two for the $S_{A}$ curve. As suggested in reference 1 it is possible to obtain either pure absorptive or pure dispersive signal by setting the frequency to, respectively, the $C_{A}$ zero or one of the $S_{A}$ zeros. Thus, changes in the two ultrasonic parameters a and $v$ may be monitored independently.

The theoretical points plotted in these figures show excellent agreement with the experimental curves. The deviations that do occur seem to be explained by sample inhomogeneity. It is concluded then, that the theory of the one-dimensional isolated resonator correctly predicts the sensitivity enhancement line shapes. 


\section{CONCLUDING REMARKS}

The sensitivity enhancement line shape factors which occur in analysis of ultrasonic resonator systems have been measured. The experimental values concur with predictions based on the isolated resonator theory. Calculations of the sensitivity enhancement line shapes associated with the acoustic signals $\left(|A|, A_{1}\right.$, and $\left.A_{2}\right)$ are presented along with supporting experimental figures. The experimental results were obtained with a CdS light-sensitive resonator whose acoustic properties could be modulated with a light beam. It is demonstrated how both absorptive as well as dispersive changes in the resonator can be isolated in a CdS sample. Furthermore, a technique is described to obtain pure absorptive or dispersive signals by use of the zero crossings of the resonator line shapes.

\section{Langley Research Center,}

National Aeronautics and Space Administration, Hampton, Va., October 30, 1973. 


\section{REFERENCES}

1. Miller, J. G.; and Bolef, D. I.: Sensitivity Enhancement by the Use of Acoustic Resonators in cw Ultrasonic Spectroscopy. J. Appl. Phys., vol. 39, no. 10, Sept. 1968, pp. $4589-4593$.

2. Bolef, D. I.; and Miller, J. G.: High-Frequency Continuous Wave Ultrasonics. Physical Acoustics, vol. VIII, Warren P. Mașon and R. W. Thurston, eds., Academic Press, Inc., 1971, pp. 95-201.

3. Miller, J. G.; and Bolef, D. I.: Sampled-cw Study of "Inhomogeneous" Ultrasonic Responses in Solids. J. Appl. Phys., vol. 41, no. 6, May 1970, pp. 2282-2293.

4. Miller, J. G.; and Bolef, D. I.: A "Sampled-Continuous Wave" Ultrasonic Technique and Spectrometer. Rev. Sci. Instruments, vol. 40, no. 7, July 1969, pp. 915-920.

5. Hutson, A. R.; and White, Donald L.: Elastic Wave Propagation in Piezoelectric Semiconductors. J. Appl. Phys., vol. 33, no. 1, Jan. 1962, pp. 40-47.

6. Kuhn, Lawrence: Measurements on Surface Acoustic Waves in Photoconducting Cadmium Sulfide. Proceedings IEE E, vol. 58, no. 9, Sept. 1970, pp. 1380-1381. 


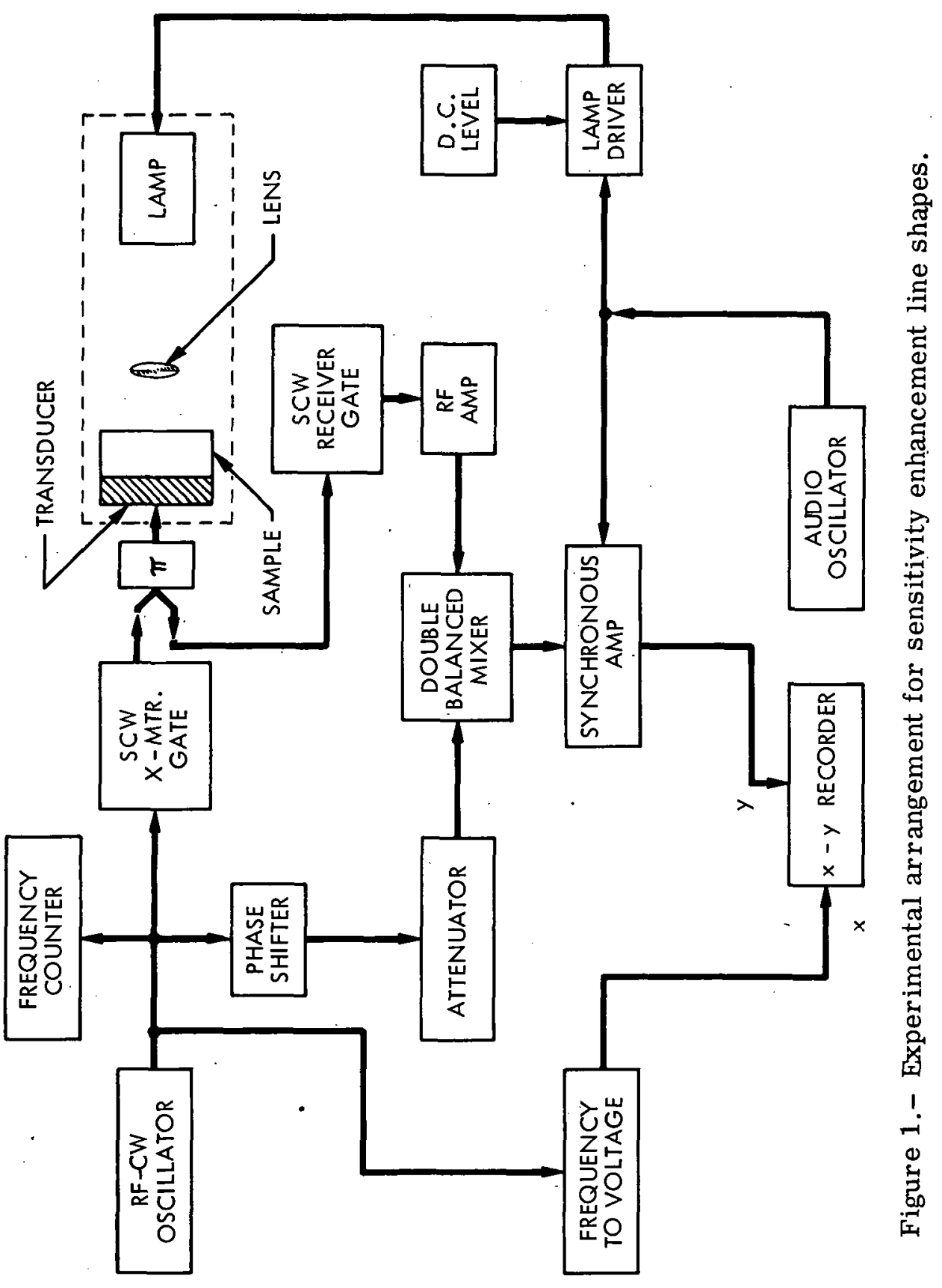




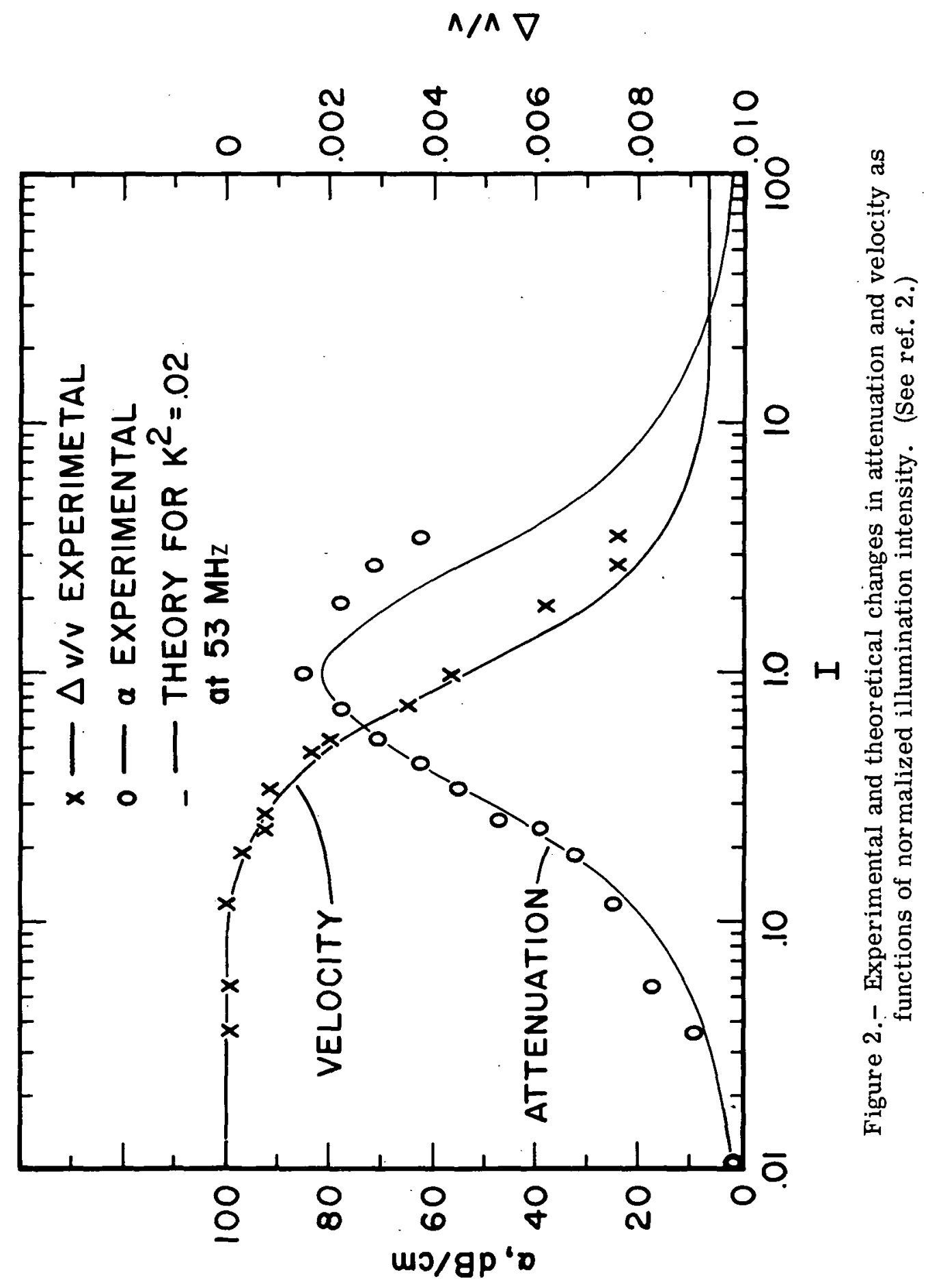




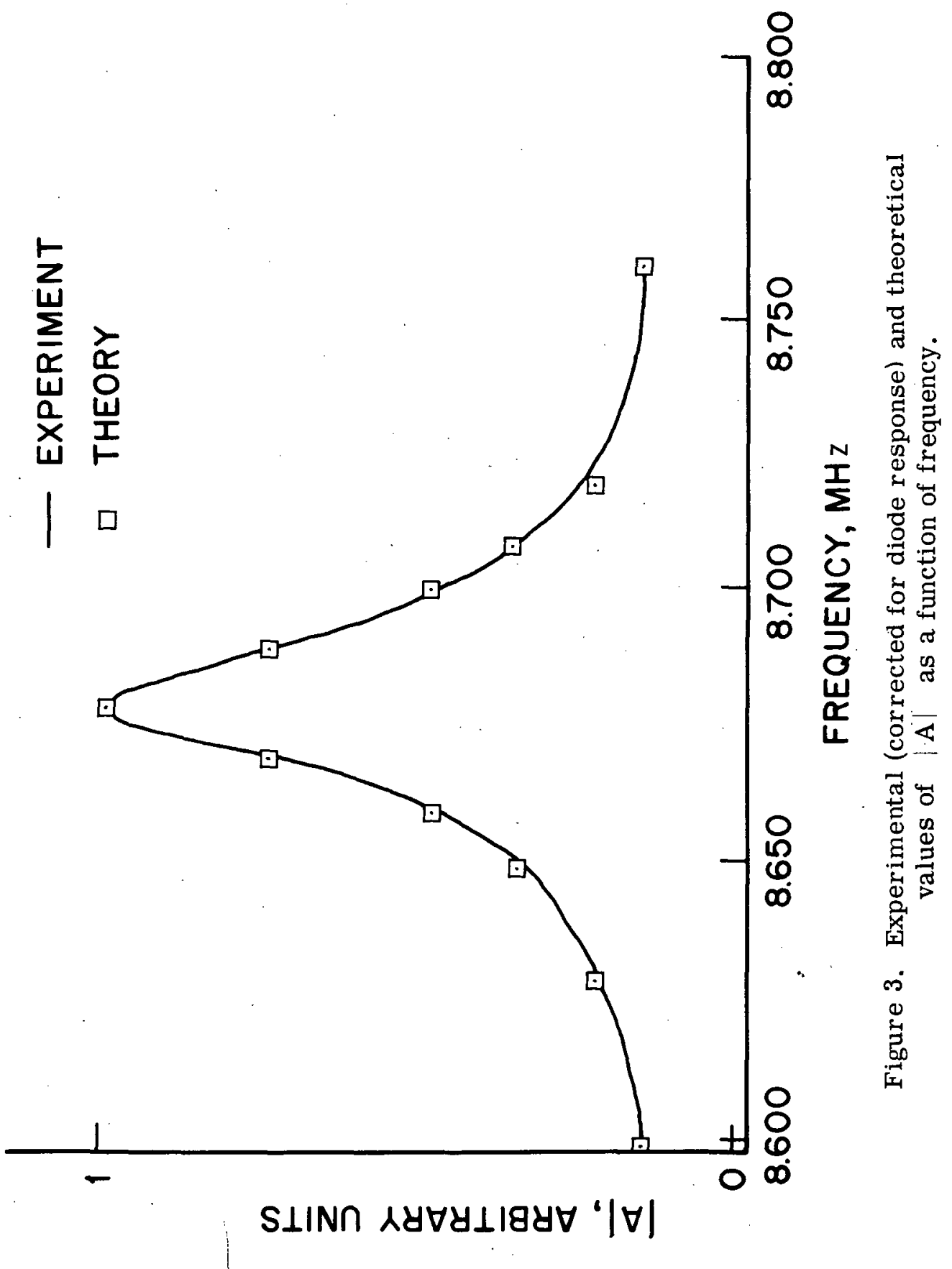




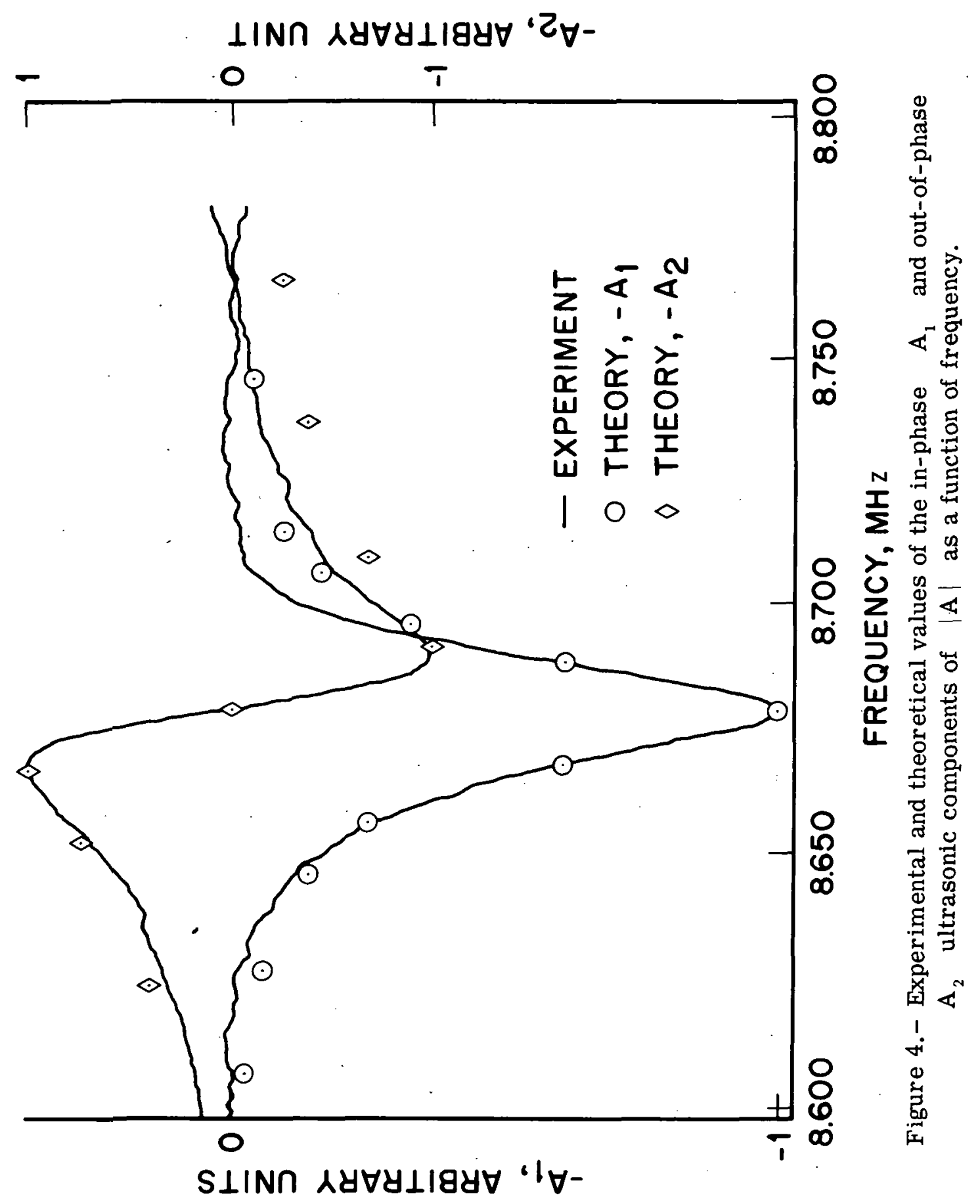




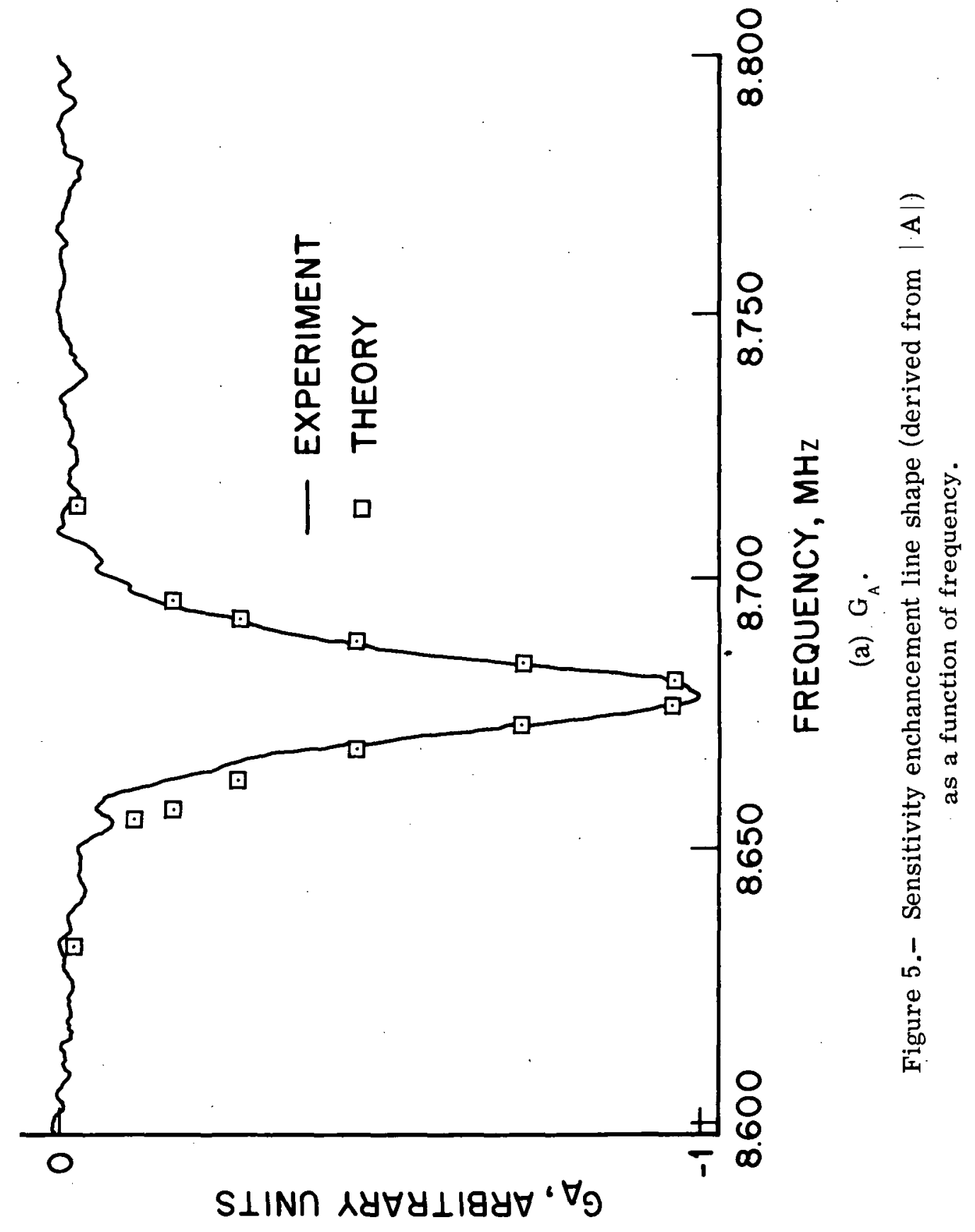




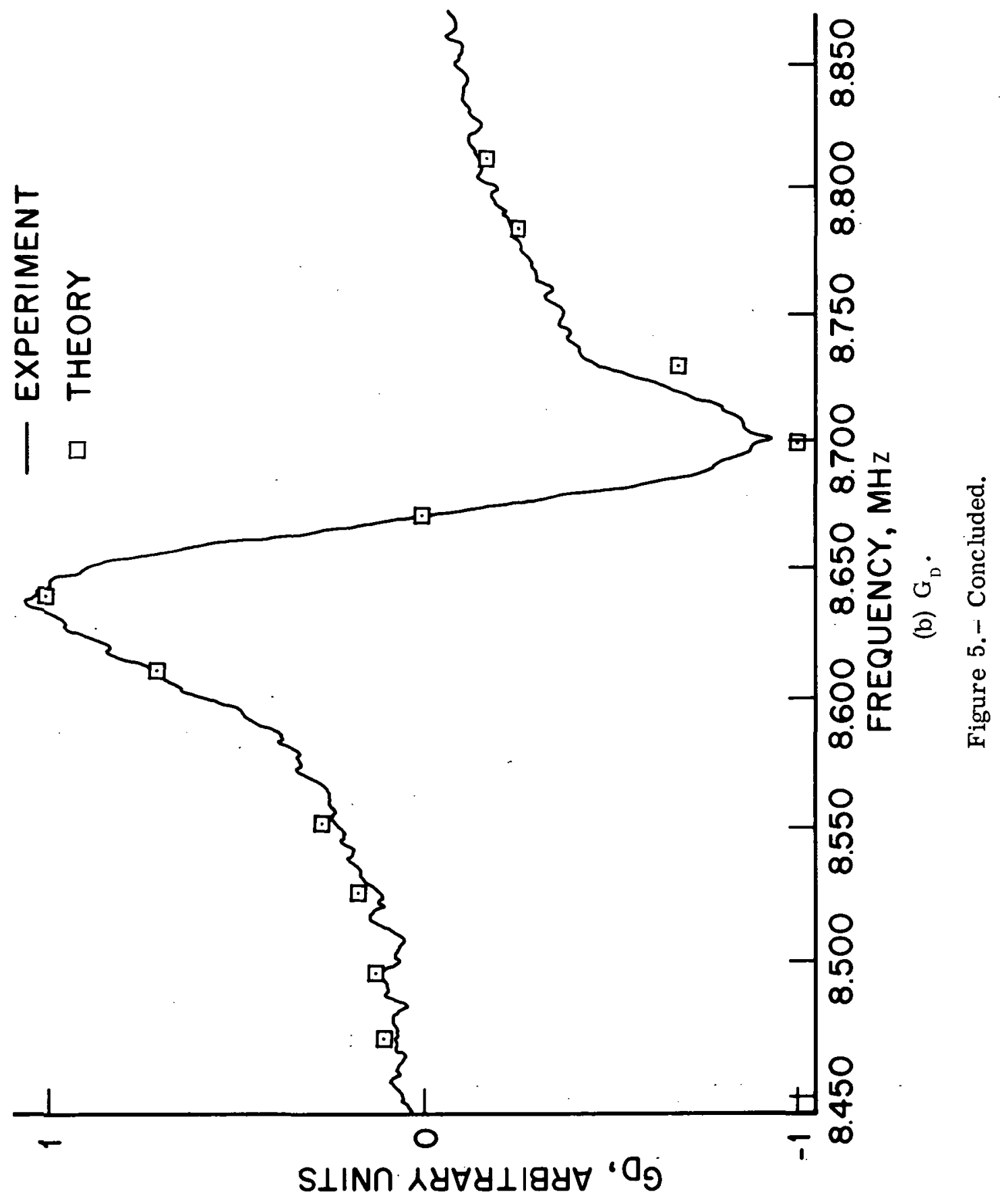




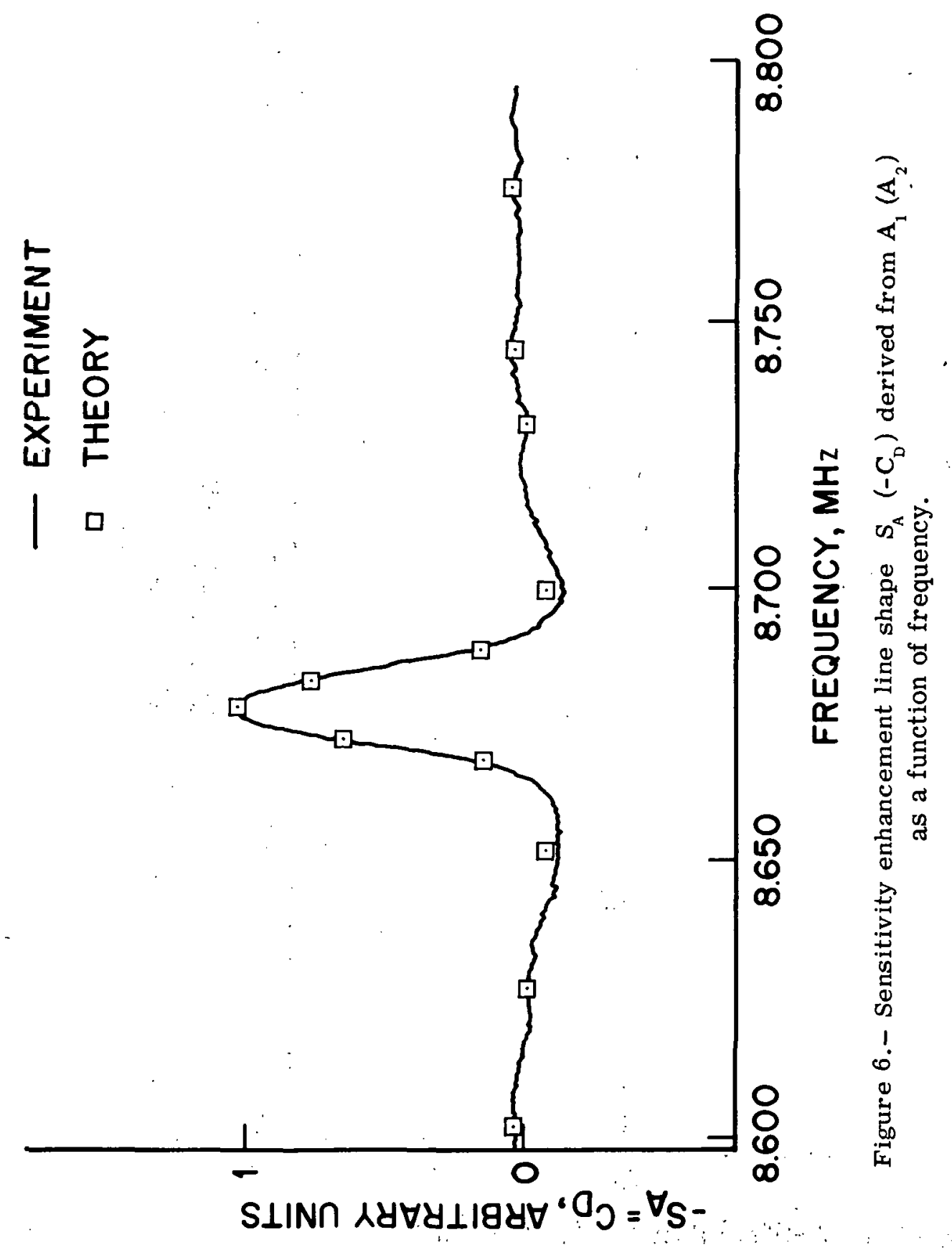




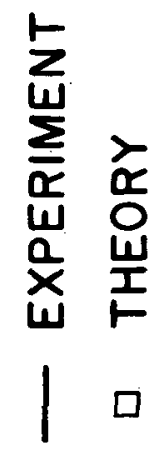

$\prod^{\infty}$

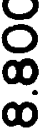

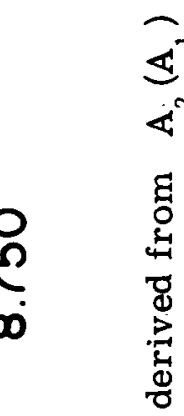

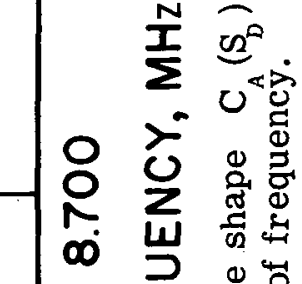

व

岃 :

品苛兽

द्वृ

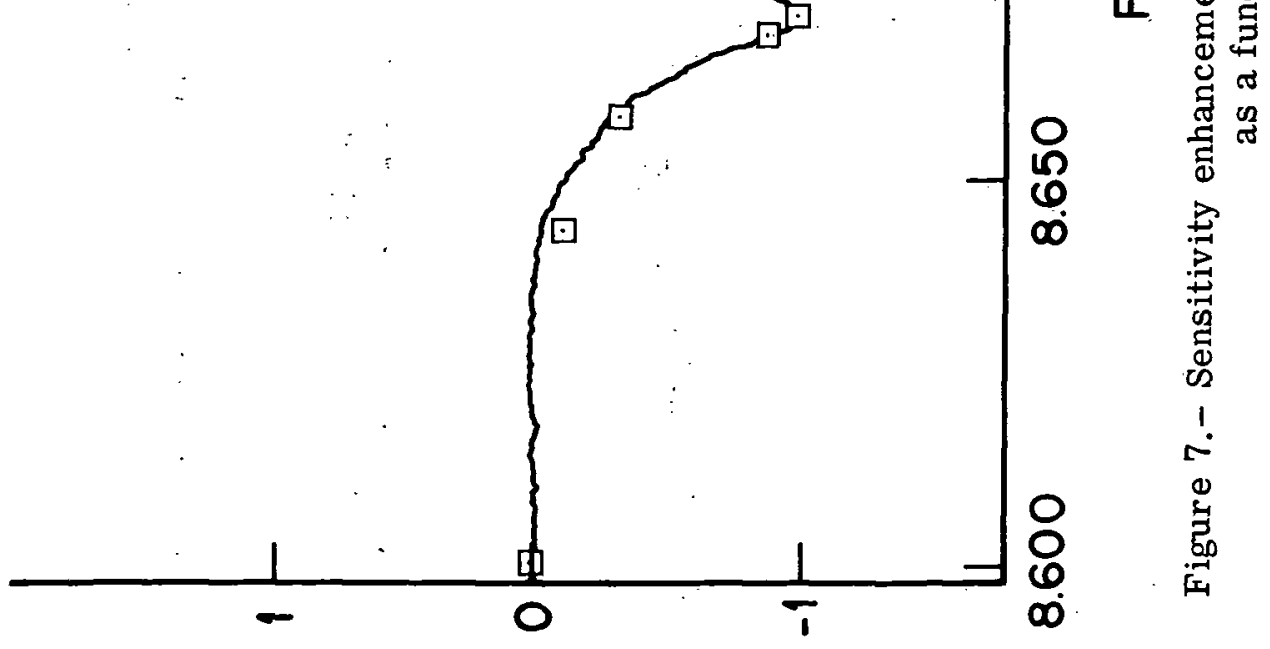

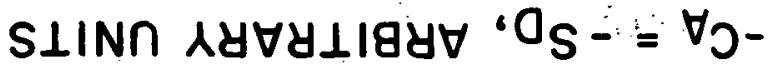


"The aeronautical and space activities of the United States shall be conducted so as to contribute... to the expansion of buman knowledge of phenomena in the atmosphere and space. The Administration shall provide for the widest practicable and appropriate dissemination of information concerning its activities and the results thereof."

\section{NASA SCIENTIFIC AND TECHNICAL PUBLICATIONS}

TECHNICAL REPORTS: Scientific and technical information considered important, complete, and a lasting contribution to existing knowledge.

TECHNICAL NOTES: Information less broad in scope but nevertheless of importance as a contribution to existing knowledge.

TECHNICAL MEMORANDUMS:

Information receiving limited distribution because of preliminary data, security classification, or other reasons. Also includes conference proceedings with either limited or unlimited distribution.

CONTRACTOR REPORTS: Scientific and technical information generated under a NASA contract or grant and considered an important contribution to existing knowledge.
TECHNICAL TRANSLATIONS: Information published in a foreign language considered to merit NASA distribution in English.

SPECIAL PUBLICATIONS: Information derived from or of value to NASA activities. Publications include final reports of major projects, monographs, data compilations, handbooks, sourcebooks, and special bibliographies.

\section{TECHNOLOGY UTILIZATION}

PUBLICATIONS: Information on technology used by NASA that may be of particular interest in commercial and other non-aerospace applications. Publications include Tech Briefs, Technology Utilization Reports and Technology Surveys.

Defails on the availability of these publications may be obtained from:

SCIENTIFIC AND TECHNICAL INFORMATION OFFICE 